\title{
Femotosecond switching in a dual-core-fiber nonlinear coupler
}

\author{
S. R. Friberg, * A. M. Weiner, Y. Silberberg, B. G. Sfez, ${ }^{\dagger}$ and P. S. Smith \\ Bell Communications Research, 331 Newman Springs Road, Red Bank, New Jersey, 07701-7020
}

Received April 25, 1988; accepted June 22, 1988

\begin{abstract}
We report all-optical switching of 100 -fsec pulses in a fused-quartz dual-core-fiber directional coupler. The length of the device is $0.5 \mathrm{~cm}$, and the switching power is $32 \mathrm{~kW}$. Pulses are routed to either of two separate fiber guides, depending on the input power. Measurements of pulse reshaping by the nonlinear coupler provide compelling evidence of the device's ability to response on a femotosecond time scale.
\end{abstract}

One reason for the current upsurge of interest in photonic switching has been the realization that all-optical devices have the capability to switch at rates much higher than those possible with electronics technology. Such high rates are likely to be necessary in future high-speed communications and computing systems. ${ }^{1}$

Optical glasses have a number of advantages as nonlinear materials for all-optical devices. Their high transparency permits long interaction lengths in guided-wave structures and essentially eliminates the thermal heating problems that have affected the performance of all-optical devices made from semiconductor and nonlinear organic materials. ${ }^{2}$ Certain optical glasses have the highest figure of merit of any nonlinear-optical material. ${ }^{2}$

In the past few years, a number of glass optical-fiber switching devices have been demonstrated, including the birefringent-fiber polarization switch, ${ }^{3}$ the optical-fiber Kerr gate, ${ }^{4-6}$ and the two-core-fiber directional coupler. 7,8 For the coupler, a signal is routed between two waveguides; for the polarization switch and the Kerr gate, a signal is transferred between polarization modes in a single waveguide. In this Letter we report substantially complete switching of 100 fsec optical pulses by a glass-fiber nonlinear coupler. We believe this to be the fastest switching time ever measured in a guided-wave all-optical device.

A nonlinear coupler, shown schematically in Fig. 1(A), consists of two closely spaced, parallel, singlemode waveguides in a material with an intensity-dependent index of refraction. At low light levels, the device behaves as a linear directional coupler. Because of evanescent coupling, signals introduced into guide (1) transfer completely to guide (2) in one coupling length $L_{c}$. Higher intensities induce changes in the refractive index and detune the coupler. Coupling is inhibited for input powers above the critical power $P_{c}=A \lambda / n_{2} L_{c}$, where $A$ is the effective mode area, $\lambda$ is the vacuum wavelength, and $n_{2}$ is the nonlinear index. ${ }^{9}$ A nonlinear coupler of length $L_{c}$ exhibits particularly useful switching characteristics. The solid curves in Fig. 1(B) show the calculated fractional power emerging from each of waveguides (1) and (2) as a function of input power, for a constant-intensity input signal. ${ }^{9}$ Low-power signals introduced into guide (1) emerge from guide (2), whereas high-power signals ( $P$ $\gg P_{c}$ ) emerge from guide (1).

Our nonlinear coupler consists of a 5.0-mm length of dual-core fused-quartz optical fiber. The fiber contains two $2.8-\mu \mathrm{m}$-diameter, Ge-doped cores with 8.4 $\mu \mathrm{m}$ between core centers and with a core-cladding index difference of 0.003 . Each fiber core is single mode for wavelengths longer than $500 \mathrm{~nm}$. We determined the coupling length by using a low-power whitelight measurement technique, in which a tunable monochromatic source is focused into a single fiber core. The relative output of each fiber core was measured over a wavelength range of $500-800 \mathrm{~nm}$, and the

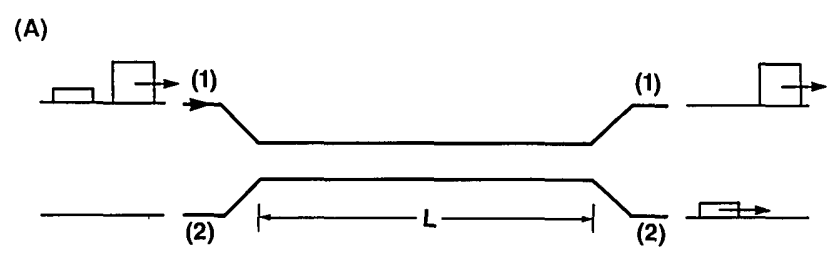

(B)

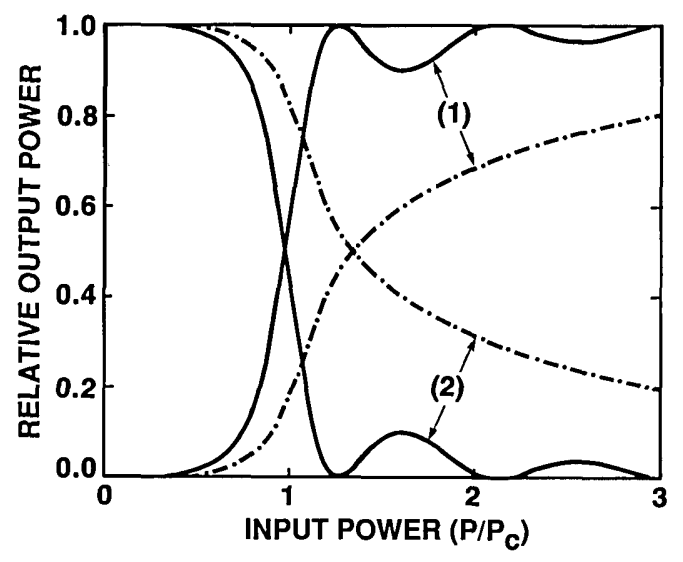

Fig. 1. (A) Schematic of a nonlinear coupler. (B) Calculated fractional output power emerging from waveguides (1) and (2) as a function of input power, for a coupler of length $L_{c}$. The input power is normalized to $P_{c}$. The curves were computed from Eq. (26) of Ref. 8. Solid curves: constantintensity input signal. Dashed-dotted curves: coupler response integrated over a $\operatorname{sech}^{2}(t)$ pulse intensity profile. 


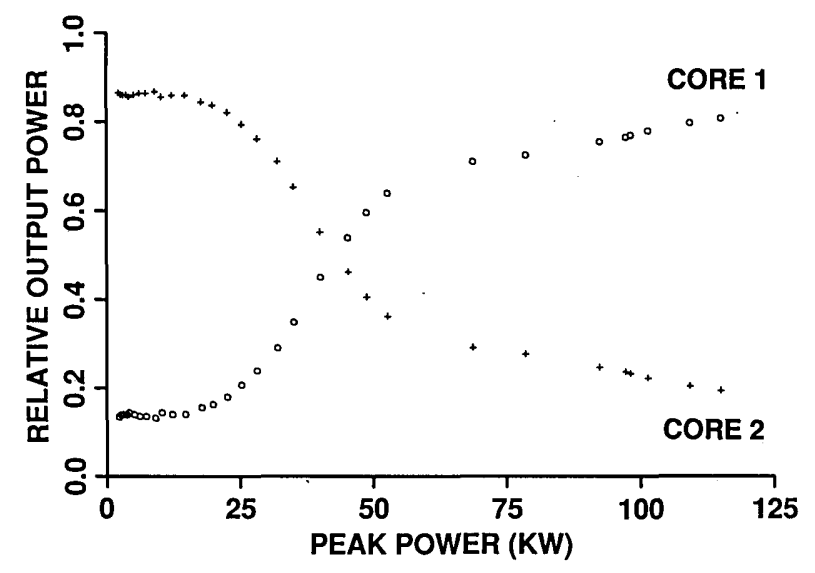

Fig. 2. Fractional output power from waveguides (1) and (2) for the 5-mm, dual-core-fiber nonlinear coupler. These data are the response for 100 -fsec input pulses.

results were fitted to a theoretical model of the coupler. From this procedure, we estimate a coupling length of $4.7 \mathrm{~mm}$ at $620 \mathrm{~nm}$. As part of the low-power characterization, we verified that the coupling length was independent of polarization and that the coupler maintained the polarization state of the input light.

Switching experiments were performed using pulses from a colliding-pulse mode-locked dye laser and a copper-vapor-laser pumped dye amplifier system. ${ }^{10}$ The laser produced 100 -fsec pulses at a wavelength of $620 \mathrm{~nm}$, which were amplified at an $8.6-\mathrm{kHz}$ repetition rate to $100 \mathrm{~nJ}$. When desired, longer pulses could be obtained by adjustment of the dispersion-compensating prisms within the laser. Amplified pulses were focused by a $20 \times$ microscope objective into one fiber core, which we denote guide (1); the other input core was carefully blocked by the edge of a razor blade. An attenuator wheel was used to control the input intensity to the fiber. The output from each core was focused by a $40 \times$ microscope objective onto a separate power meter, and the average power emerging from each core was measured as a function of the input power. The output from each core could be directed to a real-time autocorrelator to monitor the pulse shape or to a 0.32 $\mathrm{m}$ spectrometer and photodiode array to observe the power spectrum.

The fraction of the average output power emerging from each guide is plotted in Fig. 2 as a function of peak power. Similar data were obtained for several pieces of dual-core fiber, for various laser pulse widths in the 100-200-fsec range, and for various polarization angles. Because the power meters respond slowly compared to the pulse duration, we expect to measure not the cw response curves shown in Fig. 1(B) but rather a response integrated over the pulse's intensity profile. At high power the data are in good agreement with the theoretical integrated response function [dashed-dotted curves in Fig. 1(B)], calculated for a coupler of length $L_{c}$ and a sech ${ }^{2}$ intensity profile. From the data we determine a critical power $P_{c}=32$ $\mathrm{kW}$, about a factor of 2 lower than the $60-\mathrm{kW}$ value obtained from the formula $P_{c}=A \lambda / n_{2} L_{c}$, using the known nonlinear coefficient for silica $\left(n_{2}=3.2 \times 10^{-16}\right.$ $\mathrm{cm}^{2} / \mathrm{W}$ ) and using $15 \mu \mathrm{m}^{2}$ as a rough estimate of the effective mode area. Because of the difficulty in accurately measuring the peak power of femtosecond pulses and because of the uncertainty in the effective mode area, we consider that this discrepancy is within our experimental limits. At low power the data depart from theory and indicate incomplete energy transfer from one guide to the other. We attribute this result to partial excitation of radiation modes that are not completely extinguished in the 5 -mm length of fiber.

Output pulses from the nonlinear coupler can be strongly reshaped. ${ }^{11}$ For peak powers above $P_{c}$, the intense central portion of the input pulse emerges from guide (1), whereas the output of guide (2) is a pulse doublet corresponding to the low-intensity wings of the input. This is confirmed by autocorrelation measurements of pulses emerging from the nonlinear coupler. At low powers, the pulses emerging from guides (1) and (2) are identical. Autocorrelations obtained for 200fsec pulses at a power of $\simeq 2 P_{c}$ are plotted in Figs. 3(A) and $3(\mathrm{~B})$, respectively, for guides (1) and (2). While the pulses from guide (1) are similar to those at low power, the pulses from guide (2) are strongly reshaped. The triply peaked autocorrelation trace corresponds to a doubly peaked intensity profile, and the 340 -fsec peak separation is consistent with the duration of the input pulse. Further, we note that the individual peaks are significantly narrower than the input. These data show that for input powers $>P_{c}$ the coupler slices out the central part of the input pulse and directs it to the other output port. Our data show that both on and off switching times are $<100$ fsec.

In addition to nonlinear reshaping, intense pulses propagating in a nonlinear coupler are spectrally and temporally broadened by self-phase modulation and group-velocity dispersion. For peak input powers below $0.5 P_{c}$, we observe that the spectra are unbroadened, with a width of $10 \mathrm{~nm}$ FWHM. For higher powers the spectra broaden continuously, doubling to $20 \mathrm{~nm}$ at $\simeq 1.3 P_{c}$. As the power is raised further, spectra corresponding to pulses emerging from guide (2) become increasingly complex, whereas the spectra

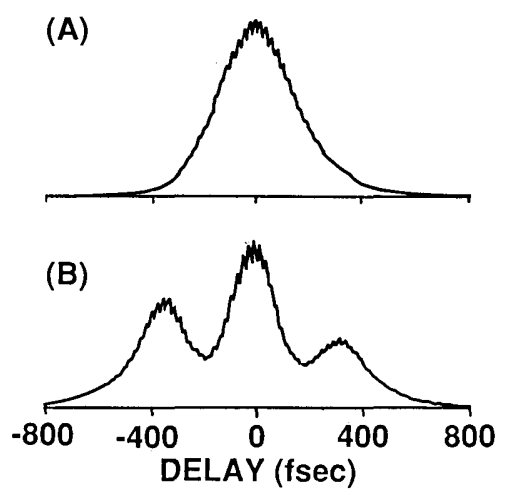

Fig. 3. Autocorrelation traces for output pulses from the coupler. The input pulses were $200 \mathrm{fsec}$ in duration. (A) Guide (1), peak power $P \simeq 2 P_{c}$. (B) Guide (2), peak power $P$ $\simeq 2 P_{c}$. The asymmetry in the trace results from a slight misalignment of the autocorrelator. 
of pulses emerging from guide (1) remain relatively unstructured. The temporal broadening of pulses emerging from guide (1) can easily be estimated if we neglect nonlinear reshaping effects. In that case we must consider pulse propagation under the combined influence of self-phase modulation and group-velocity dispersion; this topic has been analyzed in detail in the literature. ${ }^{12}$ For a dispersion of $0.265 \mathrm{psec} / \mathrm{nm}-\mathrm{m}$ at our 620 -nm wavelength, a $100-$ fsec pulse with peak power $P_{c}$ would broaden by $\simeq 50 \%$ at the fiber output; a 200 -fsec pulse at $P_{c}$ would broaden by $\simeq 25 \%$. Temporal broadening within the nonlinear coupler would normally be expected to limit the maximum bit rate attainable with the device. At a wavelength of $1.3 \mu \mathrm{m}$, the group-velocity dispersion is zero; other factors, such as the wavelength dependence of the coupling length, would determine the maximum bit rate.

During the course of our experiments, we observed that prolonged exposure to high-intensity illumination modified the coupler operation. Specifically, after exposure to peak powers of $\simeq 100 \mathrm{~kW}$ (i.e., $\simeq 3 P_{c}$ ) for a period of a few minutes, the fraction of light coupled at low power from guide (1) to guide (2) decreased, typically by several percent, although the switching behavior was not noticeably affected. Significantly longer exposures caused a decrease by as much as $25 \%$ in the low-power coupling ratio and an increase in switching power. Annealing the coupler for $4 \mathrm{~h}$ at $600^{\circ} \mathrm{C}$ restored the original response. Our observations are consistent with previous observations of persistent photoinduced refractive-index changes in Ge-doped fibers ${ }^{13,14}$; a persistent index change could cause a mismatch between the two fiber cores and inhibit coupling. We expect that photosensitivity effects may be reduced by using fibers with pure fused-quartz cores ${ }^{14}$ and by operating at longer wavelengths. ${ }^{15}$

The performance of the nonlinear coupler could be enhanced in several ways. The switching power could be reduced by using a longer device fabricated from a more highly nonlinear material. For example, a 20$\mathrm{cm}$ device fabricated from SF-59 glass (which has a nonlinear refractive index 25 times higher than that of fused quartz ${ }^{2}$ ) should switch at $\simeq 30 \mathrm{~W}$. Decreasing the fiber-core size would reduce the switching power further and bring it closer to the range accessible to mode-locked diode lasers. Operation of the coupler with ultrashort square pulses ${ }^{16}$ would lead to further enhancements: measurements would demonstrate sharper switching transitions and would avoid incomplete energy transfer owing to pulse fragmentation.

In conclusion, we have demonstrated switching of 100 -fsec pulses in a $0.5-\mathrm{cm}$ dual-core-fiber nonlinear coupler. To our knowledge this is the fastest switching ever observed in a waveguide all-optical switching device.

We thank E. Snitzer of Polaroid Corporation for the dual-core fiber. We gratefully acknowledge technical assistance by D. E. Leaird and enlightening discussions with M. Saifi and W. J. Tomlinson.

* Present address, NTT Basic Research Laboratories, Nippon Telegraph and Telephone Corporation, 9-11 Midori-cho, 3-Chome, Musashino-shi, Tokyo 180 Japan.

$\dagger$ Present address, Ecole Nationale Supérieure des Télécommunications, Paris, France.

\section{References}

1. P. W. Smith, Philos. Trans. R. Soc. London Ser. A 313, 349 (1984).

2. S. R. Friberg and P. W. Smith, IEEE J. Quantum Electron. QE-23, 2089 (1987).

3. S. Trillo, S. Wabnitz, R. H. Stolen, G. Assanto, C. T. Seaton, and G. I. Stegeman, Appl. Phys. Lett. 49, 1224 (1986).

4. N. J. Halas, D. Krokel, and D. Grischkowsky, Appl. Phys. Lett. 50, 886 (1987).

5. K. Kitayama, Y. Kimura, and S. Seikai, Appl. Phys. Lett. 46, 317 (1985).

6. T. Morioka, M. Saruwatari, and A. Takeda, Electron. Lett. 23, 453 (1987).

7. S. R. Friberg, Y. Silberberg, M. K. Oliver, M. J. Andrejco, M. A. Saifi, and P. W. Smith, Appl. Phys. Lett. 52, 1135 (1987).

8. D. D. Gusovskii, E. M. Dianov, A. A. Maier, V. B. Neustruev, V. V. Osiko, A. M. Prokhorov, K. Yu. Sitarskii, and I. A. Scherbakov, Sov. J. Quantum Electron. 17, 724 (1987); A. A. Maier, Yu. N. Serdyuchenko, K. Yu. Sitarskii, M. Ya. Shchelev, and I. A. Scherbakov, Sov. J. Quantum Electron. 17, 735 (1987).

9. S. M. Jensen, IEEE J. Quantum Electron. QE-18, 1580 (1982).

10. W. H. Knox, M. C. Downer, R. L. Fork, and C. V. Shank, Opt. Lett. 9, 552 (1984).

11. K. Kitayama and S. Wang, Appl. Phys. Lett. 43, 17 (1983).

12. W. J. Tomlinson, R. H. Stolen, and C. V. Shank, J. Opt. Soc. Am. B 1, 139 (1984).

13. K. O. Hill, Y. Fujii, D. C. Johnson, and B. S. Kawasaki, Appl. Phys. Lett. 32, 647 (1978).

14. J. Stone, J. Appl. Phys. 62, 4371 (1987).

15. G. Meltz, J. R. Dunphy, W. H. Glenn, J. D. Farina, and F. J. Leonberger, Proc. Soc. Photo-Opt. Instrum. Eng. 798, 104 (1987).

16. A. M. Weiner, J. P. Heritage and R. N. Thurston, Opt. Lett. 11, 153 (1986); A. M. Weiner, J. P. Heritage, and E. M. Kirschner, J. Opt. Soc. Am. B (to be published). 